\title{
Erratum: Poisson's ratio in layered two-dimensional crystals [Phys. Rev. B 93, 075420 (2016)]
}

Sungjong Woo, Hee Chul Park, and Young-Woo Son

(Received 25 October 2016; published 12 December 2016)

DOI: 10.1103/PhysRevB.94.239901

In plotting multiple Young's moduli for graphene, $h$-BN, and $\mathrm{MoS}_{2}$ simultaneously in Figs. 2(a) and 2(c) of our paper, Young's moduli for $\mathrm{MoS}_{2}$ along the in-plane $\left(E_{i}\right)$ and out-of-plane $\left(E_{o}\right)$ direction are not properly converted from atomic units to GPa. We corrected this error in Figs. 1(a) and 1(c) shown below. All the other data except $E_{i(o)}$ for MoS $\mathrm{S}_{2}$ are unchanged. We note that the corrections to the figures in no way affect any of the other results in the paper or the discussion and conclusion of the work.
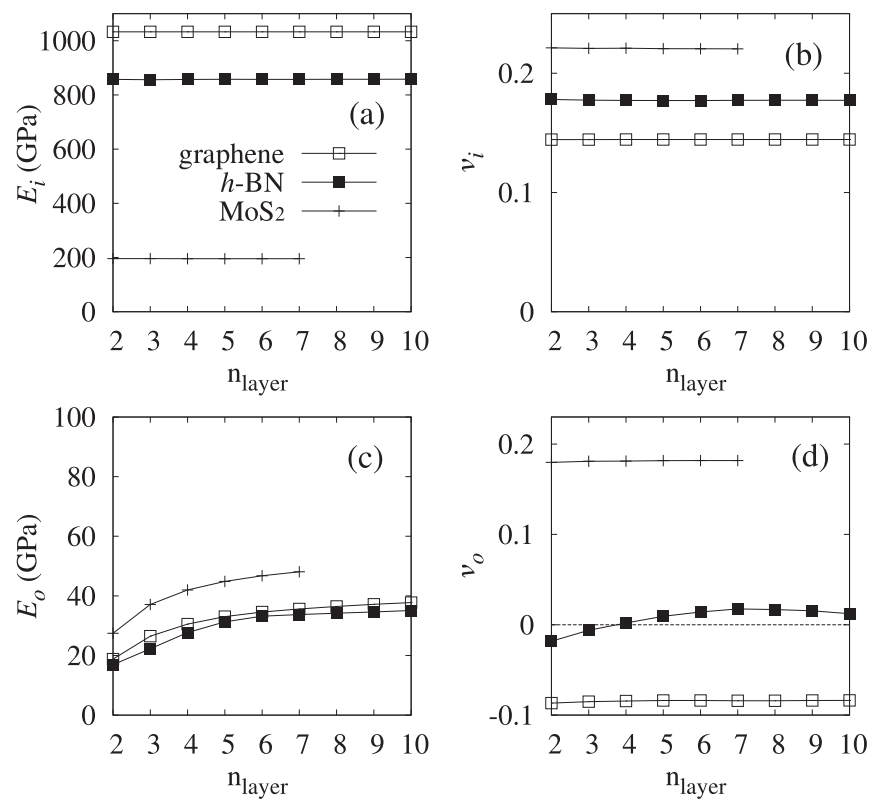

FIG. 1. Elasticity constants of graphene (empty rectangle), $h$-BN (filled rectangle), and $2 \mathrm{H}-\mathrm{MoS}_{2}$ (cross) as a function of the number of layers up to ten layers; in-plane (a) Young's modulus and (b) Poisson's ratio, and out-of-plane (c) Young's modulus and (d) Poisson's ratio. 